\title{
HOLOMORPHIC FOCK SPACES FOR POSITIVE LINEAR TRANSFORMATIONS
}

\author{
R. FABEC, G. ÓLAFSSON, and A. N. SENGUPTA*
}

\begin{abstract}
Suppose $A$ is a positive real linear transformation on a finite dimensional complex inner product space $V$. The reproducing kernel for the Fock space of square integrable holomorphic functions on $V$ relative to the Gaussian measure $d \mu_{A}(z)=\frac{\sqrt{\operatorname{det} A}}{\pi^{n}} e^{-\operatorname{Re}\langle A z, z\rangle} d z$ is described in terms of the linear and antilinear decomposition of the linear operator $A$. Moreover, if $A$ commutes with a conjugation on $V$, then a restriction mapping to the real vectors in $V$ is polarized to obtain a Segal-Bargmann transform, which we also study in the Gaussian-measure setting.
\end{abstract}

\section{Introduction}

The classical Segal-Bargmann transform is an integral transform which defines a unitary isomorphism of $L^{2}\left(\mathrm{R}^{n}\right)$ onto the space $\mathrm{F}\left(\mathrm{C}^{n}\right)$ of entire functions on $\mathrm{C}^{n}$ which are square integrable with respect to the Gaussian measure $\mu=\pi^{-n} e^{-\|z\|^{2}} d x d y$, where $d x d y$ stands for the Lebesgue measure on $\mathrm{R}^{2 n} \simeq \mathrm{C}^{n}$, see [1], [3], [4], [5], [11], [12]. There have been several generalizations of this transform, based on the heat equation or the representation theory of Lie groups [6], [10], [13]. In particular, it was shown in [10] that the Segal-Bargmann transform is a special case of the restriction principle, i.e., construction of unitary isomorphisms based on the polarization of a restriction map. This principle was first introduced in [10], see also [9], where several examples were explained from that point of view. In short the restriction principle can be explained in the following way. Let $M_{\mathrm{C}}$ be a complex manifold and let $M \subset M_{\mathrm{C}}$ be a totally real submanifold. Let $\mathrm{F}=\mathrm{F}\left(M_{\mathrm{C}}\right)$ be a Hilbert space of holomorphic functions on $M_{\mathrm{C}}$ such that the evaluation maps $\mathrm{F} \ni F \mapsto F(z) \in \mathrm{C}$ are continuous for all $z \in M_{\mathrm{C}}$, i.e., $\mathrm{F}$ is a reproducing kernel Hilbert space. There exists a function $K: M_{\mathrm{C}} \times M_{\mathrm{C}} \rightarrow \mathrm{C}$ holomorphic in the first variable, anti-holomorphic in the second variable, and such that the following hold:

(a) $K(z, w)=\overline{K(w, z)}$ for all $z, w \in M_{\mathrm{C}}$;

\footnotetext{
* The research of G. Ólafsson was supported by DMS-0070607, DMS-0139473 and DMS0402068. The research of A. Sengupta was supported by DMS-0201683. The authors would like to thank the referee for valuable comments and remarks.
}

Received January 31, 2004; in revised form March 20, 2005 
(b) If $K_{w}(z):=K(z, w)$ then $K_{w} \in \mathrm{F}$ and

$$
F(w)=\left\langle F, K_{w}\right\rangle_{\mathrm{F}}, \quad \forall F \in \mathrm{F}, z \in M_{\mathrm{C}} .
$$

The function $K$ is the reproducing kernel for the Hilbert space F. Let $D$ : $M \rightarrow \mathrm{C}^{*}$ be measurable. Then the restriction map $R: F \mapsto R F:=\left.D F\right|_{M}$ is injective. Assume that there is a measure $\mu$ on $M$ such that $R F \in L^{2}(M, \mu)$ for all $F$ in a dense subset of $\mathrm{F}$. Assuming that $R$ is closeable, $\operatorname{Im}(R)$ is dense in $L^{2}(M, \mu)$, and by polarizing $R^{*}$, we can write

$$
R^{*}=U\left|R^{*}\right|
$$

where $U: L^{2}(M, \mu) \rightarrow \mathrm{F}$ is a unitary isomorphism and $\left|R^{*}\right|=\sqrt{R R^{*}}$. Using the fact that $F$ is a reproducing kernel Hilbert space we get

$$
U f(z)=\left\langle U f, K_{z}\right\rangle_{\mathrm{F}}=\left\langle f, U^{*} K_{z}\right\rangle_{L^{2}}=\int_{M} f(m) \overline{\left(U^{*} K_{z}\right)(m)} d \mu(m) .
$$

Thus $U$ is always an integral operator. We notice also that the formula for $U$ shows that the important object in this analysis is the reproducing kernel $K(z, w)$.

We will use the following notation through this article: Let $\langle z, w\rangle=z_{1} \overline{w_{1}}+$ $\cdots+z_{n} \overline{w_{n}}$ be the standard inner product on $\mathrm{C}^{n}$ and let $(z, w)=\operatorname{Re}(\langle z, w\rangle)$ be the corresponding inner product on $\mathrm{C}^{n}$ viewed as a $2 n$-dimensional real vector spaces. Notice that $(x, y)=\langle x, y\rangle=x_{1} y_{1}+\cdots+x_{n} y_{n}$ for $x, y \in \mathrm{R}^{n}$. We write $z^{2}=z_{1}^{2}+\cdots+z_{n}^{2}$ for $z \in \mathrm{C}^{n}$.

The reproducing kernel for the classical Fock space is given by $K(z, w)=$ $e^{\langle z, w\rangle}$, where $z, w \in \mathrm{C}^{n}$. By taking $D(x):=(2 \pi)^{-n / 4} e^{-x^{2} / 2}$, for $x \in \mathrm{R}^{n}$, which is closely related to the heat kernel, we arrive at the classical Segal-Bargmann transform, given as the holomorphic continuation of

$$
U g(x)=(2 / \pi)^{n / 4} e^{x^{2} / 2} \int_{\mathrm{R}^{n}} g(y) e^{-(x-y)^{2}} d y .
$$

Notice that $\mathbf{R}^{n} \ni x \mapsto U g(x) \in \mathbf{C}$ has a unique holomorphic extension to $\mathrm{C}^{n}$.

The same principle can be used to construct the Hall-transform for compact Lie groups, [6]. In [2], Driver and Hall, motivated by application to quantum Yang-Mills theory, introduced a Fock space and Segal-Bargmann transform depending on two parameters $r, s>0$, giving different weights to the $x$ and $y$ directions, where $z=x+i y \in \mathrm{C}^{n}$ (this was also studied in [13]). Thus $\mathrm{F}$ is now the space of holomorphic functions $F(z)$ on $\mathrm{C}^{n}$ which are square-integrable with respect to the Gaussian measure $d M_{r, s}(z)=\frac{1}{(\pi r)^{n / 2}(\pi s)^{n / 2}} e^{-\frac{x^{2}}{r}-\frac{y^{2}}{s}} d x d y$. A Segal-Bargmann transform for this Fock space is given in [13] and in Theorem 3 
of [7]. We show this is a very special case of a larger family of Fock spaces and associated Segal-Bargmann tranforms. Indeed, if $A$ is a real linear positive definite matrix $A$ on a complex inner product space $V$, then

$$
d \mu_{A}(z)=\frac{\sqrt{\operatorname{det}(A)}}{\pi^{n}} e^{-(A z, z)}|d z|
$$

gives rise to a Fock space $F_{A}$. We find an expression for the reproducing kernel $K_{A}(z, w)$. We use the restriction principle to construct a natural generalization of the Segal-Bargmann transform for this space, with a certain natural restriction on $A$. We study this also in the Gaussian setting, and indicate a generalization to infinite dimensions.

We will fix the following notation for the types of bilinear pairings that we shall be using in this paper:

(i) $\langle z, w\rangle$ denotes a Hermitian inner product on a complex vector space $V$, i.e., a pairing which is complex-linear in $z$, complex-conjugate-linear in $w$, and $\langle z, z\rangle>0$ if $z \neq 0$. We denote by $\|z\|=\sqrt{\langle z, z\rangle}$ the corresponding norm;

(ii) $(x, y)$ denotes an inner product on $V$ viewed as a real vector space. The standard choice is $(x, y)=\operatorname{Re}\langle x, y\rangle$;

(iii) $z \cdot w$ denotes a complex-bilinear pairing. In the standard situation we have $z \cdot w=\langle z, \bar{w}\rangle$. We set $z^{2}=z \cdot z$.

\section{The Fock space and the restriction principle}

In this section we recall some standard facts about the classical Fock space of holomorphic functions on $C^{n}$. We refer to [5] for details and further information. Let $\mu$ be the measure $d \mu(z)=\pi^{-n} e^{-\|z\|^{2}} d x d y$ and let $\mathrm{F}$ be the classical Fock-space of holomorphic functions $F: \mathrm{C}^{n} \rightarrow \mathrm{C}$ such that

$$
\|F\|_{\mathrm{F}}^{2}:=\int_{\mathrm{C}^{n}}|F(z)|^{2} d \mu(z)<\infty .
$$

The space $F$ is a reproducing Hilbert space with inner product

$$
\langle F, G\rangle_{\mathrm{F}}=\int_{\mathrm{C}^{n}} F(z) \overline{G(z)} d \mu(z)
$$

and reproducing kernel $K(z, w)=e^{\langle z, w\rangle}$.

Thus

$$
F(w)=\int_{\mathrm{C}^{n}} F(z) \overline{K(z, w)} d \mu(z)=\left\langle F, K_{w}\right\rangle_{\mathrm{F}}
$$


where $K_{w}(z)=K(z, w)$. The function $K(z, w)$ is holomorphic in the first variable, anti-holomorphic in the second variable, and $K(z, w)=\overline{K(w, z)}$. Notice that $K(z, z)=\left\langle K_{z}, K_{z}\right\rangle_{\mathrm{F}}$. Hence $\left\|K_{z}\right\|_{\mathrm{F}}=e^{\|z\|^{2} / 2}$. Finally the linear space of finite linear combinations $\sum c_{j} K_{z_{j}}, z_{j} \in \mathrm{C}^{n}, c_{j} \in \mathrm{C}$, is dense in $\mathrm{F}$. An orthonormal system in $\mathrm{F}$ is given by the monomials $e_{\alpha}(z)=z_{1}^{\alpha_{1}} \cdots z_{n}^{\alpha_{n}} / \sqrt{\alpha_{1} ! \cdots \alpha_{n} !}$, $\alpha \in \mathrm{N}_{0}^{n}$. If the reference to the Fock space is clear, then we simply write $\langle\cdot, \cdot\rangle$ instead of $\langle\cdot, \cdot\rangle_{\mathrm{F}}$, and similarly for the corresponding norm.

View $\mathbf{R}^{n} \subset \mathrm{C}^{n}$ as a totally real submanifold of $\mathrm{C}^{n}$. We will now recall the construction of the classical Segal-Bargmann transform using the restriction principle, see [9], [10]. For constructing a restriction map as explained in the introduction we need to choose the function $D(x)$. One motivation for the choice of $D$ is the heat kernel, but another one, more closely related to representation theory, is that the restriction map should commute with the action of $\mathbf{R}^{n}$ on the Fock space and $L^{2}\left(\mathbf{R}^{n}\right)$. Indeed, take

$$
T(x) F(z)=m(x, z) F(z-x)
$$

for $F$ in $\mathrm{F}$ where $m(x, z)$ has properties sufficient to make $x \mapsto T(x)$ a unitary representation of $\mathbf{R}^{n}$ on $\mathrm{F}$. Namely, we need a multiplier $m$ satisfying

$$
|m(x, z)|=\left(\frac{d \mu(z-x)}{d \mu(z)}\right)^{\frac{1}{2}}=e^{\left(\operatorname{Re}(z, x\rangle-x^{2} / 2\right)} .
$$

We take $m(x, z):=e^{\langle z, x\rangle-x^{2} / 2}$. Set

$$
D(x)=(2 \pi)^{-n / 4} m(0, x)=(2 \pi)^{-n / 4} e^{-x^{2} / 2}
$$

and define $R: \mathrm{F} \rightarrow C^{\infty}\left(\mathrm{R}^{n}\right)$ by

$$
R F(x):=D(x) F(x)=(2 \pi)^{-n / 4} e^{-x^{2} / 2} F(x) .
$$

Then

$$
R T(y) F(x)=R F(x-y) .
$$

Since $F$ is holomorphic, the map $R$ is injective. Furthermore, the holomorphic polynomials $p(z)=\sum a_{\alpha} z^{\alpha}$ are dense in $\mathrm{F}$ and obviously $R p \in L^{2}\left(\mathrm{R}^{n}\right)$. Thus, we may and will consider $R$ as a densely defined operator from $\mathrm{F}$ into $L^{2}\left(\mathrm{R}^{n}\right)$. The Hermite functions $h_{\alpha}(x)=(-1)^{|\alpha|}\left(D^{\alpha} e^{-\|x\|^{2}}\right) e^{x^{2} / 2}$ are images under $R$ of polynomials and thus are in the image of the operator $R$. Hence, $\operatorname{Im}(R)$ is dense in $L^{2}\left(\mathrm{R}^{n}\right)$. Using continuity of the evaluation maps $F \mapsto F(z)$, it can be checked that $R$ is a closed operator. Hence, $R$ has a densely-defined adjoint 
$R^{*}: L^{2}\left(\mathrm{R}^{n}\right) \rightarrow \mathrm{F}$. For $z, w \in \mathrm{C}^{n}$, recall that $z \cdot w=\sum z_{j} w_{j}$. Then, for any $g$ in the domain of $R^{*}$, we have:

$$
\begin{aligned}
R^{*} g(z)=\left\langle R^{*} g, K_{z}\right\rangle=\left\langle g, R K_{z}\right\rangle & =(2 \pi)^{-n / 4} \int_{\mathrm{R}^{n}} g(y) e^{-\|y\|^{2} / 2} e^{z \cdot y} d y \\
& =(2 \pi)^{-n / 4} e^{z^{2} / 2} \int_{\mathrm{R}^{n}} g(y) e^{-(z-y)^{2} / 2} d y \\
& =(2 \pi)^{n / 4} e^{z^{2} / 2} g * p(z)
\end{aligned}
$$

where $p(z)=(2 \pi)^{-n / 2} e^{-z^{2} / 2}$ is holomorphic. Applying the map $R: \mathbf{F} \rightarrow$ $C^{\infty}\left(\mathrm{R}^{n}\right)$, we have

$$
R R^{*} g(x)=g * p(x) .
$$

Since $p \in L^{1}\left(\mathrm{R}^{n}\right)$ and $g \in L^{2}\left(\mathrm{R}^{n}\right)$, it follows that $g * p \in L^{2}\left(\mathrm{R}^{n}\right)$, and so the preceding equation shows that $R^{*} g$ is in the domain of the operator $R$, and so $g$ is in the domain of the operator composite $R R^{*}$. This argument also shows that $R R^{*}$ is a bounded operator, on its domain, with operator norm $\left\|R R^{*}\right\|\|p\|_{1}$. Moreover, for every $g$ in the domain of $R^{*}$, we have

$$
\left\langle R^{*} g, R^{*} g\right\rangle=\left\langle R R^{*} g, g\right\rangle \leq\left\|R R^{*}\right\|\|g\|_{2} .
$$

Thus $R^{*}$ is a bounded operator. Being an adjoint, it is also closed. Therefore, the domain of $R^{*}$ is in fact the full space $L^{2}\left(\mathrm{R}^{n}\right)$. So for any $f \in D(R)$, we have

$$
\langle R f, R f\rangle=\left\langle R^{*}(R f), f\right\rangle \leq\left\|R^{*}\right\|\|R f\|_{2}\|f\|_{2},
$$

which implies that the operator $R$ is bounded. Again, being a closed, denselydefined bounded operator, $R$ is, in fact, defined on all of $\mathrm{F}$. In summary,

Lemma 1.1. The linear operators $R$ and $R^{*}$ are everywhere defined and continuous.

Let $p_{t}(x)=(2 \pi t)^{-n / 2} e^{-x^{2} / 2 t}$ be the heat kernel on $\mathrm{R}^{n}$. Then $\left(p_{t}\right)_{t>0}$ is a convolution semigroup and $p=p_{1}$. Hence $\sqrt{R R^{*}}=p_{1 / 2} *$ or

$$
R U g(x)=\left|R^{*}\right| g(x)=p_{1 / 2} * g(x)=\pi^{-n / 2} \int_{\mathrm{R}^{n}} g(y) e^{-(x-y)^{2}} d y .
$$

It follows that

$$
U g(x)=(2 / \pi)^{n / 4} e^{x^{2} / 2} \int_{\mathrm{R}^{n}} g(y) e^{-(x-y)^{2}} d y
$$


for $x \in \mathrm{R}^{n}$. But the function on the right hand side is holomorphic in $x$. Analytic continuation gives the following classical Segal-Bargmann tranform.

THEOREM 1.2. The map $U: L^{2}\left(\mathrm{R}^{n}\right) \rightarrow \mathrm{F}$ given by

$$
U g(z)=(2 / \pi)^{n / 4} \int_{\mathrm{R}^{n}} g(y) \exp \left(-y^{2}+2\langle z, y\rangle-z^{2} / 2\right) d y
$$

is a unitary isomorphism.

\section{Twisted Fock spaces}

Let $V \simeq \mathrm{C}^{n}$ be a finite dimensional complex vector space of complex dimension $n$ and let $\langle\cdot, \cdot\rangle$ be a complex Hermitian inner-product.

We will also consider $V$ as a real vector space with real inner product defined by $(z, w)=\operatorname{Re}\langle z, w\rangle$. Notice that $(z, z)=\langle z, z\rangle$ for all $z \in \mathrm{C}^{n}$. Let $J$ be the real linear transformation of $V$ given by $J z=i z$. Note that $J^{*}=-J=J^{-1}$ and thus $J$ is a skew symmetric real linear transformation. Fix a real linear transformation $A$. Then $A=H+K$ where

$$
H:=\frac{A+J^{-1} A J}{2} \quad \text { and } \quad K:=\frac{A-J^{-1} A J}{2} .
$$

We have $H J=\frac{1}{2}\left(A J-J^{-1} A\right)=\frac{1}{2} J\left(J^{-1} A J+A\right)=J H$ and $K J=$ $\frac{1}{2}\left(A J+J^{-1} A\right)=\frac{1}{2} J\left(J^{-1} A J-A\right)=-J K$. Thus $H$ is complex linear and $K$ is conjugate linear.

We now assume that $A$ is symmetric and positive definite relative to the real inner product $(\cdot, \cdot)$.

Lemma 2.1. The complex linear transformation $H$ is self adjoint, positive with respect to the inner product $\langle\cdot, \cdot\rangle$, and invertible.

Proof. Since $A$ is positive and invertible as a real linear transformation, we have $(A z, z)>0$ for all $z \neq 0$. But $J$ is real linear and skew symmetric. Hence $\left(J A J^{-1} z, z\right)>0$ for all $z \neq 0$. In particular $H=\frac{1}{2}\left(A+J A J^{-1}\right)$ is complex linear, symmetric with respect to the real inner product $(\cdot, \cdot)$, and positive. Consequently $\operatorname{Re}\langle H i v, w\rangle=\operatorname{Re}\langle i v, H w\rangle$. This implies $\operatorname{Im}\langle H v, w\rangle=$ $\operatorname{Im}\langle v, H w\rangle$ and hence $\langle H v, w\rangle=\langle v, H w\rangle$. Thus $H$ is complex self adjoint and $\langle H z, z\rangle>0$ for $z \neq 0$.

Lemma 2.2. Let $w \in V$. Then $\langle A w, w\rangle=(A w, w)+i \operatorname{Im}\langle K w, w\rangle$ and $(A w, w)=(H w, w)+(K w, w)$.

Proof. The first statement follows from

$$
\begin{aligned}
\langle A w, w\rangle=\langle H w, w\rangle+\langle K w, w\rangle & =(H w, w)+(K w, w)+i \operatorname{Im}\langle K w, w\rangle \\
& =(A w, w)+i \operatorname{Im}\langle K w, w\rangle .
\end{aligned}
$$


Taking the real part in the second line gives the second claim, which also follows directly from bilinearity of $(\cdot, \cdot)$.

Denote by $\operatorname{det}_{\mathrm{R}}(\cdot)$ the determinant of a R-linear map on $V \simeq \mathrm{C}^{n} \simeq \mathrm{R}^{2 n}$ and by $\operatorname{det}(\cdot)$ the determinant of a complex linear map of $V$. Let $\mu_{A}$ be the measure defined by $d \mu_{A}(z)=\pi^{-n} \sqrt{\operatorname{det}_{\mathrm{R}} A} e^{-(A z, z)} d x d y$ and let $\mathrm{F}_{A}$ be the space of holomorphic functions $F: \mathrm{C}^{n} \rightarrow \mathrm{C}$ such that

$$
\|F\|_{A}^{2}:=\int|F(z)|^{2} d \mu_{A}(z)<\infty .
$$

Our normalization of $\mu$ is chosen so that $\|1\|_{A}=1$. Just as in the classical case one can show that $F_{A}$ is a reproducing kernel Hilbert space, but this will also follow from the following Lemma. We notice that all the holomorphic polynomials $p(z)$ are in $\mathrm{F}$. To simplify the notation, we let $T_{1}=H^{-1 / 2}$. Then $T_{1}$ is symmetric, positive definite and complex linear. Let $c_{A}=\sqrt{\operatorname{det}_{\mathrm{R}}\left(A^{1 / 2} T_{1}\right)}=$ $\left(\operatorname{det}_{\mathrm{R}}(A) / \operatorname{det}_{\mathrm{R}}(H)\right)^{1 / 4}$.

Lemma 2.3. Let $F: V \rightarrow \mathrm{C}$ be holomorphic. Then $F \in \mathrm{F}_{A}$ if and only if $F \circ T_{1} \in \mathrm{F}$. Moreover, the map $\Psi: \mathrm{F}_{A} \rightarrow \mathrm{F}$ given by

$$
\Psi(F)(w):=c_{A} \exp \left(-\overline{\left\langle K T_{1} w, T_{1} w\right\rangle} / 2\right) F\left(T_{1} w\right)
$$

is a unitary isomorphism. In particular

$$
\Psi^{*} F(w)=\Psi^{-1} F(w)=c_{A}^{-1} \exp (\overline{\langle K w, w\rangle} / 2) F(\sqrt{H} w) .
$$

Proof. Note $F$ is holomorphic if and only if $F \circ T_{1}$ is holomorphic as $T_{1}$ is complex linear and invertible. Moreover, unitarity follows from

$$
\begin{aligned}
\|\Psi F\|^{2} & =\pi^{-n} \int_{V}|\Psi F(w)|^{2} e^{-\langle w, w\rangle} d w \\
& =\pi^{-n} \sqrt{\operatorname{det}_{\mathrm{R}} A} \int_{V}|F(w)|^{2} e^{-(K w, w)} e^{-\langle\sqrt{H} w, \sqrt{H} w\rangle} d w \\
& =\pi^{-n} \sqrt{\operatorname{det}_{\mathrm{R}} A} \int_{V}|F(w)|^{2} e^{-(K w, w)} e^{-\langle H w, w\rangle} d w \\
& =\pi^{-n} \sqrt{\operatorname{det}_{\mathrm{R}} A} \int_{V}|F(w)|^{2} e^{-((H+K) w, w)} d w \\
& =\pi^{-n} \sqrt{\operatorname{det}_{\mathrm{R}} A} \int_{V}|F(w)|^{2} e^{-(A w, w)} d w \\
& =\|F\|_{A}^{2} .
\end{aligned}
$$


THEOREM 2.4. The space $\mathrm{F}_{A}$ is a reproducing kernel Hilbert space with reproducing kernel

$$
K_{A}(z, w)=c_{A}^{-2} e^{\frac{1}{2} \overline{\langle K z, z\rangle}} e^{\langle H z, w\rangle} e^{\frac{1}{2}\langle K w, w\rangle} .
$$

Proof. By Lemma 2.3 we get

$$
\begin{aligned}
c_{A} \exp \left(-\overline{\left\langle K T_{1} w, T_{1} w\right\rangle} / 2\right) F\left(T_{1} w\right) & =\Psi(F)(w) \\
& =\left(\Psi(F), K_{w}\right)_{\mathrm{F}} \\
& =\left(F, \Psi^{*}\left(K_{w}\right)\right)_{\mathrm{F}_{A}} .
\end{aligned}
$$

Hence

$$
K_{A}(z, w)=c_{A}^{-1} \exp (\overline{\langle K w, w\rangle} / 2) \Psi^{*}\left(K_{\sqrt{H} w}\right)=c_{A}^{-2} e^{\frac{1}{2} \overline{\langle K z, z\rangle}} e^{\langle H z, w\rangle} e^{\frac{1}{2}\langle K w, w\rangle} .
$$

\section{The Restriction Map}

We continue to assume $A>0$. We notice that Lemma 2.3 gives a unitary isomorphism $\Psi^{*} U: L^{2}\left(\mathrm{R}^{n}\right) \rightarrow \mathrm{F}_{A}$, where $U$ is the classical Segal-Bargmann transform. But this is not the natural transform that we are looking for. As $H$ is positive definite there is an orthonormal basis $e_{1}, \ldots, e_{n}$ of $V$ and positive numbers $\lambda_{j}>0$ such that $H e_{j}=\lambda_{j} e_{j}$. Let $V_{\mathrm{R}}:=\sum \operatorname{R} e_{k}$. Set $\sigma\left(\sum a_{i} e_{i}\right)=$ $\sum \bar{a}_{i} e_{i}$. Then $\sigma$ is a conjugation with $V_{\mathrm{R}}=\{z: \sigma z=z\}$. For $z \in V$ we will when convenient write $\bar{z}$ for $\sigma(z)$. We say that a vector is real if it belongs to $V_{\mathrm{R}}$. As $H e_{j}=\lambda_{j} e_{j}$ with $\lambda_{j} \in \mathrm{R}$ it follows that $H V_{\mathrm{R}} \subseteq V_{\mathrm{R}}$. We note that for the complex linear mapping $H$ that $\operatorname{det}_{\mathrm{R}} H=(\operatorname{det} H)^{2}$ and that $\operatorname{det} H$ is equal to the determinant of the real linear transformation $\left.H\right|_{V_{\mathrm{R}}}$.

Lemma 3.1. $\langle K z, w\rangle=\langle K w, z\rangle$.

Proof. Note that $\sigma K$ is complex linear. Since $J^{*}=-J, K=\frac{1}{2}(A-$ $\left.J A J^{-1}\right)$ is real symmetric. Thus $(K w, z)=(w, K z)=(K z, w)$. Also note

$$
(i K z, w)=(J K z, w)=-(K J z, w)=-(J z, K w)=-(i z, K w) .
$$

Hence $\operatorname{Re}\langle i K z, w\rangle=-\operatorname{Re}\langle i z, K w\rangle$. So $-\operatorname{Im}\langle K z, w\rangle=\operatorname{Im}\langle z, K w\rangle$. This gives $\operatorname{Im}\langle K w, z\rangle=\operatorname{Im}\langle K z, w\rangle$. Hence $\langle K z, w\rangle=\langle K w, z\rangle$.

LeMma 3.2. $(\sigma K)^{*}=K \sigma$.

Proof. We have $\langle\sigma z, \sigma w\rangle=\langle w, z\rangle$. Hence

$$
\langle\sigma K z, w\rangle=\left\langle\sigma w, \sigma^{2} K z\right\rangle=\langle\sigma w, K z\rangle=\langle z, K \sigma w\rangle .
$$


Corollary 3.3. If $x, y \in V_{\mathrm{R}}$, then $\langle H x, y\rangle$ is real and $\langle A x, y\rangle=\langle A y, x\rangle$.

Proof. Clearly $\langle\cdot, \cdot\rangle$ is real on $V_{\mathrm{R}} \times V_{\mathrm{R}}$. Since $H V_{\mathrm{R}} \subseteq V_{\mathrm{R}}$, we see $\langle H x, y\rangle$ is real. Next, $\langle A x, y\rangle=\langle H x, y\rangle+\langle K x, y\rangle$. The term $\langle H x, y\rangle$ equals $\langle H y, x\rangle$ because $\langle H x, y\rangle$ is real and $H$ is self-adjoint. On the other hand, $\langle K x, y\rangle=$ $\langle K y, x\rangle$ by Lemma 3.1. So $\langle A x, y\rangle=\langle A y, x\rangle$.

As before we would like to have a multiplier $m: V_{\mathrm{R}} \times V \rightarrow \mathrm{C}^{*}$ such that

$$
T(x) F(z)=m(x, z) F(z-x)
$$

is a unitary representation of $\mathrm{F}_{A}$ that commutes with translation on $L^{2}\left(V_{\mathrm{R}}\right)$. It turns out the multipliers we construct are co-boundaries under translation by elements of $V_{\mathrm{R}}$ on $V$.

Definition 3.4. A function $m$ is a co-boundary on $V \cong \mathrm{C}^{n}$ under translation by $V_{\mathrm{R}}$ if there is a nonzero complex valued function $b$ on $V$ with

$$
m(x, z)=b(z-x) b(z)^{-1} \quad \text { for } x \in V_{\mathrm{R}} \text { and } z \in V .
$$

It is well known and easy to verify that every co-boundary $m$ on $V$ under translation by $V_{\mathrm{R}}$ is a multiplier.

Lemma 3.5. The function

$$
m(x, z)=e^{\langle H z, x\rangle} e^{\langle K \bar{z}, x\rangle} e^{-\langle A x, x\rangle / 2}=e^{\langle A x, \bar{z}\rangle-\langle A x, x\rangle / 2}
$$

is a co-boundary.

Proof. Define $b(z)=e^{-\langle H z+K \bar{z}, \bar{z}\rangle / 2}$. Then

$$
\begin{aligned}
b(z-x) b(z)^{-1} & =e^{-\langle H(z-x)+K(\bar{z}-x), \bar{z}-x\rangle / 2} e^{\langle H z+K \bar{z}, \bar{z}\rangle / 2} \\
& =e^{\langle\langle H x+K x, \bar{z}\rangle+\langle H z+K \bar{z}, x\rangle) / 2} e^{-\langle H x+K x, x\rangle / 2} \\
& =e^{\langle H z, x\rangle} e^{\langle K \bar{z}, x\rangle} e^{-\langle A x, x\rangle / 2} \\
& =e^{\langle H x, \bar{z}\rangle+\langle K x, \bar{z}\rangle} e^{-\langle A x, x\rangle / 2} \\
& =e^{\langle A x, \bar{z}\rangle-\langle A x, x\rangle / 2}
\end{aligned}
$$

since $A=H+K,\langle H x, \bar{z}\rangle=\langle z, \sigma H x\rangle=\langle z, H x\rangle=\langle H z, x\rangle$, and $\langle K x, \bar{z}\rangle=$ $\langle\sigma \bar{z}, \sigma K x\rangle=\langle K \sigma z, x\rangle=\langle K \bar{z}, x\rangle$.

Corollary 3.6. Let $m(x, z)=e^{\langle A x, \bar{z}\rangle-\langle A x, x\rangle / 2}$. Set $T_{x} F(z):=m(x, z) F(z$ $-x)$ for $x \in V_{\mathrm{R}}$. Then $x \mapsto T_{x}$ is a representation of the abelian group $V_{\mathrm{R}}$ on $\mathrm{F}_{A}$. It is unitary if and only if $K V_{\mathrm{R}} \subseteq V_{\mathrm{R}}$, or equivalently $A V_{\mathrm{R}} \subseteq V_{\mathrm{R}}$. 
Proof. Since $m$ is a multiplier, we have $T_{x} T_{y}=T_{x+y}$. For each $T_{x}$ to be unitary, we need $|m(x, z)|=e^{(A z, x)-(A x, x) / 2}$. But

$$
|m(x, z)|=e^{(H z, x)} e^{(K \bar{z}, x)} e^{-(A x, x) / 2}=e^{(A z, x)-(A x, x) / 2} e^{(K \bar{z}-K z, x)} .
$$

Thus $T_{x}$ is unitary for all $x$ if and only if the real part of every vector $K \bar{z}-K z$ is 0 . Since $\bar{z}-z$ runs over $i V_{\mathrm{R}}$ as $z$ runs over $V, T_{x}$ is unitary for all $x$ if and only if $K\left(i V_{\mathrm{R}}\right) \subset i V_{\mathrm{R}}$, which is equivalent to $K\left(V_{\mathrm{R}}\right) \subset V_{\mathrm{R}}$. But since $A=H+K$ and $H$ leaves $V_{\mathrm{R}}$ invariant, this is equivalent to $V_{\mathrm{R}}$ being invariant under $A$.

REMARK. There is no uniqueness in the choice of a real vector space $V_{R}$ such that $H V_{\mathrm{R}} \subseteq V_{\mathrm{R}}$ and $V=V_{\mathrm{R}} \oplus i V_{\mathrm{R}}$. Indeed, any orthonormal basis $e_{1}, e_{2}, \ldots, e_{n}$ of eigenvectors for $H$ gives such a subspace. But since $A$ is only real linear on $V$, an interesting question is when one can choose $V_{\mathrm{R}}$ with $A V_{\mathrm{R}} \subseteq V_{\mathrm{R}}$, and in this case how unique is the choice of $V_{\mathrm{R}}$ ? This probably depends on the degree of non complex linearity of the tranformation $A$.

Recall that $\operatorname{det}_{\mathrm{R}} H=(\operatorname{det} H)^{2}$. To simplify some calculations later on we define $c:=(2 \pi)^{-n / 4}\left(\frac{\operatorname{det}_{\mathrm{R}} A}{\operatorname{det} H}\right)^{1 / 4}$. We remark for further reference:

Lemma 3.7. $c_{A}^{-2} c^{2}=\frac{\sqrt{\operatorname{det} H}}{(2 \pi)^{n / 2}}$ and $c^{-1} \frac{\sqrt{\operatorname{det}(H)}}{\pi^{n / 2}}=\left(\frac{2}{\pi}\right)^{n / 4} \frac{(\operatorname{det} H)^{3 / 4}}{\left(\operatorname{det}_{R} A\right)^{1 / 4}}$.

Let $D(x)=c m(x, 0)=c e^{-\langle A x, x\rangle / 2}$ and define $R: \mathrm{F}_{A} \rightarrow C^{\infty}\left(V_{\mathrm{R}}\right)$ by

$$
R F(x):=D(x) F(x) .
$$

Since $m$ is holomorphic on $V^{2}, D$ has a holomorphic extension to $V$.

LemMa 3.8. The restriction map $R$ intertwines the action of $V_{R}$ on $F_{A}$ and the left regular action $L$ on functions on $V_{R}$.

Proof. For all $x, y \in V_{\mathrm{R}}$, we have

$$
\begin{aligned}
R\left(T_{y} F\right)(x) & =c m(x, 0) T_{y} F(x) \\
& =c m(x, 0) m(y, x) F(x-y) \\
& =c m(x, 0) m(-y,-x) F(x-y) \\
& =c m(x-y, 0) F(x-y) \\
& =L_{y} R F(x) .
\end{aligned}
$$

\section{The Generalized Segal-Bargmann Transform}

As for the classical space, $R$ specifies a densely defined closed operator $\mathrm{F}_{A} \rightarrow$ $L^{2}\left(V_{\mathrm{R}}\right)$. It also has dense image in $L^{2}\left(V_{\mathrm{R}}\right)$. To see this, let $\left\{h_{\alpha}\right\}_{\alpha}$ be the orthonormal basis of $L^{2}\left(V_{\mathrm{R}}\right)$ given by the Hermite functions. Then $\left\{\operatorname{det}(A)^{\frac{1}{4}} h_{\alpha}(\sqrt{A} x)\right\}_{\alpha}$ 
is an orthonormal basis of $L^{2}\left(V_{R}\right)$ which is contained in the image of the set of polynomial functions under $R$. It follows again that $R$ has a densely defined adjoint and

$$
R^{*} h(z)=\left\langle R^{*} h, K_{A, z}\right\rangle=\left\langle h, R K_{A, z}\right\rangle
$$

where $K_{A, z}(w)=K_{A}(w, z)=c_{A}^{-2} e^{\frac{1}{2} \overline{\langle K w, w\rangle}} e^{\langle H w, z\rangle} e^{\frac{1}{2}\langle K z, z\rangle}$. Thus

$$
\begin{aligned}
& R^{*} h(z)=c \int h(x) e^{-\langle A x, x\rangle / 2} \overline{K_{A}(x, z)} d x \\
& =c_{A}^{-2} c \int h(x) e^{-\langle A x, x\rangle / 2} e^{\frac{1}{2} \overline{\langle K z, z\rangle}} e^{\langle z, H x\rangle} e^{\frac{1}{2}\langle K x, x\rangle} d x \\
& =c_{A}^{-2} c e^{\frac{1}{2} \overline{\langle K z, z\rangle}} \int h(x) e^{-\langle H x, x\rangle / 2} e^{-\langle K x, x\rangle / 2} e^{\langle z, H x\rangle} e^{\frac{1}{2}\langle K x, x\rangle} d x \\
& =c_{A}^{-1} c e^{\frac{1}{2} \overline{\langle K z, z\rangle}} \int h(x) e^{-\langle x, H x\rangle / 2} e^{\langle z, H x\rangle} d x \\
& =c_{A}^{-2} c e^{\frac{1}{2} \overline{K z, z\rangle}} e^{\frac{1}{2}\langle z, H \bar{z}\rangle} \int h(x) e^{-(\langle z, H \bar{z}\rangle-\langle z, H x\rangle-\langle x, H \bar{z}\rangle+\langle x, H x\rangle) / 2} d x \\
& =c_{A}^{-2} c e^{\frac{1}{2} \overline{2}\langle\bar{z}, z\rangle} e^{\frac{1}{2}\langle z, H \bar{z}\rangle} \int h(x) e^{-\langle z-x, H(\bar{z}-\bar{x})\rangle / 2} d x
\end{aligned}
$$

for $\langle z, H x\rangle=\langle\overline{H x}, \bar{z}\rangle=\langle H x, \bar{z}\rangle=\langle x, H \bar{z}\rangle$ and $\langle z, H x\rangle=\langle z, H \bar{x}\rangle$. Thus we finally arrive at

$$
R^{*} h(z)=c_{A}^{-2} c e^{\frac{1}{2}\langle z, H \bar{z}+K z\rangle} e^{-\frac{1}{2}\langle x, H \bar{x}\rangle} * h(z) .
$$

Let $P: V_{\mathrm{R}} \rightarrow V_{\mathrm{R}}$ be positive. Define $\phi_{P}(x)=\sqrt{\operatorname{det}(P)}(2 \pi)^{-n / 2} e^{-\|\sqrt{P} x\|^{2} / 2}$. For $t>0$, let $P(t)=\frac{1}{t} P$.

Lemma 4.1. Let the notation be as above. Then $0<t \mapsto \phi_{P(t)}$ is a convolution semigroup, i.e., $\phi_{P(t+s)}=\phi_{P(t)} * \phi_{P(s)}$.

Proof. This follows by change of parameters $y=\sqrt{P} x$ from the fact that $\phi_{\operatorname{Id}(t)}(x)=(2 \pi t)^{-n / 2} e^{-x^{2} / 2 t}$ is a convolution semigroup.

Define a unitary operator $W$ on $L^{2}\left(V_{\mathrm{R}}\right)$ by

$$
W f(x)=e^{i \operatorname{Im}\langle x, K x\rangle} f(x)=e^{i \operatorname{Im}\langle x, A x\rangle} f(x) .
$$

We see $W=I$ if $A V_{\mathrm{R}} \subseteq V_{\mathrm{R}}$ which is equivalent to the translation operators $T(x)$ being unitary.

Lemma 4.2. Let $h$ be in the domain of definition of $R^{*}$. Then

$$
R R^{*} h=W\left(\phi_{H} * h\right) .
$$


Proof. We notice first that $c_{A}^{-2} c^{2}=(2 \pi)^{-n / 2} \sqrt{\operatorname{det} H}$ by Lemma 3.7. From (4.1) we then have

$$
\begin{aligned}
R R^{*} h(x) & =c e^{-\frac{1}{2}\langle A x, x\rangle} R^{*} h(x) \\
& =c_{A}^{-2} c^{2} e^{-\frac{1}{2}\langle A x, x\rangle} e^{\frac{1}{2}\langle x, H \bar{x}+K x\rangle} e^{-\frac{1}{2}\langle y, H \bar{y}\rangle} * h(x) \\
& =(2 \pi)^{-n / 2} \sqrt{\operatorname{det}(H)} e^{-\frac{1}{2}\langle A x, x\rangle} e^{\frac{1}{2}\langle x, A x\rangle} e^{-\frac{1}{2}\langle y, H \bar{y}\rangle} * h(x) \\
& =(2 \pi)^{-n / 2} \sqrt{\operatorname{det}(H)} e^{i \operatorname{Im}\langle x, A x\rangle} \int e^{-\frac{1}{2}(y, H y)} h(x-y) d y . \\
& =(2 \pi)^{-n / 2} \sqrt{\operatorname{det}(H)} e^{i \operatorname{Im}\langle x, A x\rangle} \int e^{-\frac{\|\sqrt{H} y\|^{2}}{2}} h(x-y) d y \\
& =W\left(\phi_{H} * h\right)(x) .
\end{aligned}
$$

In the last step, we used the fact that $\phi_{H} * h \in L^{2}\left(V_{\mathrm{R}}\right)$, which follows from $\phi_{H}$ being in $L^{1}\left(V_{\mathrm{R}}\right)$ and $h \in L^{2}\left(V_{\mathrm{R}}\right)$.

Arguing as in the classical case, we see that $R$ and $R^{*}$ are everywhere defined and continuous.

Lemma 4.1 and Lemma 4.2 leads to the following corollary:

Corollary 4.3. Suppose $A V_{\mathrm{R}} \subseteq V_{\mathrm{R}}$. Then

$$
\left|R^{*}\right| h(x)=\phi_{H(1 / 2)} * h(x)=\frac{\sqrt{\operatorname{det}(H)}}{\pi^{n / 2}} \int_{V_{\mathrm{R}}} e^{-\|\sqrt{H} y\|^{2}} h(x-y) d y .
$$

Theorem 4.4 (The Segal-Bargmann Transform). Suppose A leaves $V_{\mathrm{R}}$ invariant. Then the operator $U_{A}: L^{2}\left(V_{\mathrm{R}}\right) \rightarrow \mathrm{F}_{A}$ defined by

$$
U_{A} f(z)=\left(\frac{2}{\pi}\right)^{n / 4} \frac{(\operatorname{det} H)^{3 / 4}}{\left(\operatorname{det}_{\mathrm{R}} A\right)^{1 / 4}} e^{\frac{1}{2}(\langle H z, \bar{z}\rangle+\langle z, K z\rangle)} \int e^{(H(z-y)) \cdot(z-y)} f(y) d y
$$

is a unitary isomorphism. We call the map $U_{A}$ the generalized Segal-Bargmann transform.

Proof. By polar decomposition, we can write $R^{*}=U\left|R^{*}\right|$ where $U$ : $L^{2}\left(V_{\mathrm{R}}\right) \rightarrow \mathrm{F}_{A}$ is a unitary isomorphism. Taking adjoints gives $\left|R^{*}\right| U^{*}=R$. Hence $R U=\left|R^{*}\right|$. Thus

$$
\begin{aligned}
\operatorname{cm}(x) U h(x) & =R U h(x)=\left(\left|R^{*}\right| h\right)(x) \\
& =\frac{\sqrt{\operatorname{det}(H)}}{\pi^{n / 2}} \int_{V_{\mathrm{R}}} e^{-\|\sqrt{H} y\|^{2}} h(x-y) d y .
\end{aligned}
$$


Since $m(x)=e^{-\frac{1}{2}(\langle x, H x\rangle+\langle x, K x\rangle)}$, we have using Lemma 3.7:

$$
U f(x)=\left(\frac{2}{\pi}\right)^{n / 4} \frac{(\operatorname{det} H)^{3 / 4}}{\left(\operatorname{det}_{\mathrm{R}} A\right)^{1 / 4}} e^{\frac{1}{2}(\langle x, H x\rangle+\langle x, K x\rangle)} \int e^{(x-y, H(x-y))} f(y) d y .
$$

Holomorphicity of $U f$ now implies $U f=U_{A} f$.

\section{The Gaussian Formulation}

In infinite dimensions there is no useful notion of Lebesgue measure but Gaussian measure does make sense. So, with a view to extension to infinite dimensions, we will recast our generalized Segal-Bargmann transform using Gaussian measure instead of Lebesgue measure as the background measure on $V_{\mathrm{R}}$. Of course, we have already defined the Fock space $F_{A}$ using Gaussian measure.

As before, $V$ is a finite-dimensional complex vector space with Hermitian inner-product $\langle\cdot, \cdot\rangle$, and $A: V \rightarrow V$ is a real-linear map which is symmetric, positive-definite with respect to the real inner-product $(\cdot, \cdot)=\operatorname{Re}\langle\cdot, \cdot\rangle$, i.e. $(A z, z)>0$ for all $z \in V$ except $z=0$. We assume, furthermore, that there is a real subspace $V_{\mathrm{R}}$ for which $V=V_{\mathrm{R}}+i V_{\mathrm{R}}$, the inner-product $\langle\cdot, \cdot\rangle$ is real-valued on $V_{\mathrm{R}}$, and $A\left(V_{\mathrm{R}}\right) \subset V_{\mathrm{R}}$. Denote the linear map $v \mapsto i v$ by $J$. As usual, $A$ is the sum

$$
A=H+K
$$

where $H=(A-J A J) / 2$ is complex-linear on $V$ and $K=(A+J A J) / 2$ is complex-conjugate-linear. The real subspaces $V_{\mathrm{R}}$ and $J V_{\mathrm{R}}$ are $(\cdot, \cdot)$-orthogonal because for any $x, y \in V_{\mathrm{R}}$ we have $(x, J y)=\operatorname{Re}\langle x, J y\rangle=-\operatorname{Re}(J\langle x, y\rangle)$, since $\langle x, y\rangle$ is real, by hypothesis. Since $A$ preserves $V_{\mathrm{R}}$ and is symmetric, it also preserves the orthogonal complement $J V_{\mathrm{R}}$. Thus $A$ has the block diagonal form:

$$
A=\left[\begin{array}{ll}
R & 0 \\
0 & T
\end{array}\right]=d(R, T)
$$

Here, and henceforth, we use the notation $d(X, Y)$ to mean the real-linear map $V \rightarrow V$ given by $a \mapsto X a$ and $J a \mapsto J Y a$ for all $a \in V_{\mathrm{R}}$, where $X, Y$ are real-linear operators on $V_{\mathrm{R}}$. Note that $d(X, Y)$ is complex-linear if and only if $X=Y$ and is complex-conjugate-linear if and only if $Y=-X$. The operator $d(X, X)$ is the unique complex-linear map $V \rightarrow V$ which restricts to $X$ on $V_{\mathrm{R}}$, and we denote it:

$$
X_{V}=\left[\begin{array}{ll}
X & 0 \\
0 & X
\end{array}\right]
$$

The hypothesis that $A$ is symmetric and positive-definite means that $R$ and $T$ are symmetric, positive definite on $V_{\mathrm{R}}$. Consequently, the real-linear operator $S$ on $V_{\mathrm{R}}$ given by

$$
S=2\left(R^{-1}+T^{-1}\right)^{-1}
$$


is also symmetric, positive-definite.

The operators $H$ and $K$ on $V$ are given by

$$
H=\frac{1}{2}\left(R_{V}+T_{V}\right), \quad K=d\left(\frac{1}{2}(R-T), \frac{1}{2}(T-R)\right) .
$$

Using the conjugation map

$$
\sigma: V \rightarrow V: a+i b \mapsto a-i b \quad \text { for } \quad a, b \in V_{\mathrm{R}}
$$

we can also write $K$ as

$$
K=\frac{1}{2}\left(R_{V}-T_{V}\right) \sigma
$$

Now consider the holomorphic functions $\rho_{T}$ and $\rho_{S}$ on $V$ given by

$$
\rho_{T}(z)=\frac{(\operatorname{det} T)^{1 / 2}}{(2 \pi)^{n / 2}} e^{-\frac{1}{2}\left(T_{V} z\right) \cdot z} \quad \rho_{S}(z)=\frac{(\operatorname{det} S)^{1 / 2}}{(2 \pi)^{n / 2}} e^{-\frac{1}{2}\left(S_{V} z\right) \cdot z}
$$

where $n=\operatorname{dim} V_{\mathrm{R}}$. Restricted to $V_{\mathrm{R}}$, these are density functions for Gaussian probability measures.

The Segal-Bargmann transform in this setting is given by the map

$$
S_{A}: L^{2}\left(V_{\mathrm{R}}, \rho_{S}(x) d x\right) \rightarrow \mathrm{F}_{A}: f \mapsto S_{A} f
$$

where

$$
S_{A} f(z)=\int_{V_{\mathrm{R}}} f(x) \rho_{T}(z-x) d x=\int_{V_{\mathrm{R}}} f(x) c(x, z) \rho_{S}(x) d x
$$

with $c(x, z)$ given, for $x \in V_{\mathrm{R}}$ and $z \in V$, by

$$
c(x, z)=\frac{\rho_{T}(x-z)}{\rho_{S}(x)} .
$$

It is possible to take (5.2) as the starting point, with $f \in L^{2}\left(V_{\mathrm{R}}, \rho_{S}(x) d x\right)$ and prove that: (i) $S_{A} f(z)$ is well-defined, (ii) $S_{A} f$ is in $\mathrm{F}_{A}$, (iii) $S_{A}$ is a unitary isomorphism onto $\mathrm{F}_{A}$. However, we shall not work out everything in this approach since we have essentially proven all this in the preceding sections. Full details of a direct approach would be obtained by generalizing the procedure used in [13]. In the present discussion we shall work out only some of the properties of $S_{A}$. 
Lemma 5.1. Let $w, z \in V$. Then:

(i) The function $x \mapsto c(x, z)$ belongs to $L^{2}\left(V_{\mathrm{R}}, \rho_{S}(x) d x\right)$, thereby ensuring that the integral (5.2) defining $S_{A} f(z)$ is well-defined;

(ii) The $S_{A}$-transform of $c(\cdot, w)$ is $K_{A}(\cdot, \bar{w})$ :

$$
\left[S_{A} c(\cdot, w)\right](z)=K_{A}(z, \bar{w})
$$

and so, in particular:

$$
K_{A}(z, w)=\int_{V_{\mathrm{R}}} \frac{\rho_{T}(x-z) \rho_{T}(x-\bar{w})}{\rho_{S}(x)} d x
$$

(iii) The transform $S_{A}$ preserves inner-products on the linear span of the functions $c(\cdot, w)$ :

$$
\langle c(\cdot, w), c(\cdot, z)\rangle_{L^{2}\left(V_{\mathrm{R}}, \rho_{S}(x) d x\right)}=K_{A}(w, z)=\left\langle K_{A}(\cdot, \bar{w}), K_{A}(\cdot, \bar{z})\right\rangle_{\mathrm{F}_{A}}
$$

Proof. (i) is equivalent to finiteness of $\int_{V_{\mathrm{R}}} \frac{\left|\rho_{T}(x-z)\right|^{2}}{\rho_{S}(x)} d x$, which is equivalent to positivity of the operator $2 T-S$. To see that $2 T-S$ is positive observe that

$$
\begin{aligned}
2 T-S & =2 T\left[\left(R^{-1}+T^{-1}\right)-T^{-1}\right]\left(R^{-1}+T^{-1}\right)^{-1} \\
& =2 T R^{-1}\left(R^{-1}+T^{-1}\right)^{-1}=T R^{-1} S \\
& =2\left(T^{-1}+T^{-1} R T^{-1}\right)^{-1}
\end{aligned}
$$

and in this last line $T^{-1}>0$ (since $\left.T>0\right)$ and $\left(T^{-1} R T^{-1} x, x\right)=\left(R T^{-1} x\right.$, $\left.T^{-1} x\right) \geq 0$ by positivity of $R$. Thus $2 T-S$ is positive, being twice the inverse of the positive operator $T^{-1}+T^{-1} R T^{-1}$.

(ii) Recall $v \cdot w$ for $v, w \in V$ is the symmetric complex bilinear pairing given by $v \cdot w=\langle v, \bar{w}\rangle$, and we write $v^{2}$ for $v \cdot v$. We shall denote the complexlinear operator $T_{V}$ which restricts to $T$ on $V_{\mathrm{R}}$ simply by $T$. It is readily checked that $T$ continues to be symmetric in the sense that $T v \cdot w=v \cdot T w$ for all $v, w \in V$. We start with

$$
\begin{aligned}
a & \stackrel{\text { def }}{=}\left[S_{A} c(\cdot, w)\right](z) \\
& =\int_{V_{\mathrm{R}}} \frac{\rho_{T}(x-w)}{\rho_{S}(x)} \rho_{T}(z-x) d x \\
& =(2 \pi)^{-n / 2} \frac{\operatorname{det} T}{(\operatorname{det} S)^{1 / 2}} \int_{V_{\mathrm{R}}} e^{-\frac{1}{2}[T(x-w) \cdot(x-w)+T(x-z) \cdot(x-z)-S x \cdot x]} d x \\
& =(2 \pi)^{-n / 2} \frac{\operatorname{det} T}{(\operatorname{det} S)^{1 / 2}} \int_{V_{\mathrm{R}}} e^{-\frac{1}{2}[(2 T-S) x \cdot x-2 T x \cdot(w+z)+T w \cdot w+T z \cdot z]} d x .
\end{aligned}
$$


Recall from the proof of (i) that $2 T-S>0$. For notational simplicity let $L=(2 T-S)^{1 / 2}$ and $M=L^{-1} T$. Then

$$
\begin{aligned}
a & =(2 \pi)^{-n / 2} \frac{\operatorname{det} T}{(\operatorname{det} S)^{1 / 2}} \int_{V_{\mathrm{R}}} e^{-\frac{1}{2}(L x-M(w+z))^{2}} d x e^{-\frac{1}{2}[T w \cdot w+T z \cdot z-M(w+z) \cdot M(w+z)]} \\
& =\frac{\operatorname{det} T}{(\operatorname{det} S)^{1 / 2}(\operatorname{det} L)} e^{-\frac{1}{2}[T w \cdot w+T z \cdot z-M(w+z) \cdot M(w+z)]} .
\end{aligned}
$$

To simplify the last exponent observe that by (5.4) and (5.1) we have

$$
\begin{aligned}
T w \cdot w-M w \cdot M w & =T w \cdot w-T w \cdot L^{-2} T w \\
& =T w \cdot w-T w \cdot(2 T-S)^{-1} T w \\
& =T w \cdot w-\frac{1}{2} T w \cdot\left(T^{-1}+T^{-1} R T^{-1}\right) T w \\
& =T w \cdot w-\frac{1}{2} T w \cdot\left(w+T^{-1} R w\right) \\
& =\frac{1}{2}(T w \cdot w-R w \cdot w) \\
& =-\langle K \bar{w}, \bar{w}\rangle .
\end{aligned}
$$

The same holds with $z$ in place of $w$. For the "cross term" we have

$$
\begin{aligned}
M w \cdot M z & =T w \cdot L^{-2} T z \\
& =T w \cdot(2 T-S)^{-1} T z \\
& =\frac{1}{2} T w \cdot\left(T^{-1}+T^{-1} R T^{-1}\right) T z \\
& =\frac{1}{2}(T w \cdot z+w \cdot R z) \\
& =2 w \cdot H z .
\end{aligned}
$$

Putting everything together gives

$$
\left[S_{A} c(\cdot, w)\right](z)=\frac{\operatorname{det} T}{(\operatorname{det} S)^{1 / 2}(\operatorname{det} L)} e^{\frac{1}{2}\langle K \bar{w}, \bar{w}\rangle} e^{\langle H w, \bar{z}\rangle} e^{\frac{1}{2}\langle K \bar{z}, \bar{z}\rangle} .
$$

In Lemma 6.2 below we prove that

$$
\frac{\operatorname{det} T}{(\operatorname{det} S)^{1 / 2}(\operatorname{det} L)}=\left(\frac{\operatorname{det}_{\mathrm{R}}(A)}{\operatorname{det}_{\mathrm{R}}(H)}\right)^{-1 / 2}=c_{A}^{-2} .
$$

So

$$
\left[S_{A} c(\cdot, w)\right](z)=K_{A}(w, \bar{z}) .
$$


For (iii), we have first:

$$
\langle c(\cdot, w), c(\cdot, z)\rangle_{L^{2}\left(\rho_{S}(x) d x\right)}=\left[S_{A} c(\cdot, w)\right](\bar{z})=K_{A}(\bar{z}, \bar{w})=K_{A}(w, z) .
$$

The second equality in (iii) follows since $K_{A}$ is a reproducing kernel.

\section{The evaluation map and determinant relations}

Recall

$$
K_{A}(z, w)=c_{A}^{-2} e^{\frac{1}{2}\langle z, K z\rangle+\frac{1}{2}\langle K w, w\rangle+\langle H z, w\rangle}
$$

where

$$
c_{A}^{-2}=\left(\frac{\operatorname{det}_{V} H}{\operatorname{det}_{V} A}\right)^{2}
$$

is a reproducing kernel for $\mathrm{F}_{A}$. Thus

$$
f(w)=\left\langle f, K_{A}(\cdot, w)\right\rangle=\pi^{-n} \int_{V} f(z) K_{A}(w, z)|d z|
$$

where $|d z|=d x d y$ signifies integration with respect to Lebesgue measure on the real inner-product space $V$. Thus we have

Proposition 6.1. For any $z \in V$, the evaluation map

$$
\delta_{z}: \mathrm{F}_{A} \rightarrow \mathrm{C}: f \mapsto f(z)
$$

is a bounded linear functional with norm

$$
\left\|\delta_{z}\right\|=K_{A}(z, z)^{1 / 2}=c_{A}^{-1} e^{(A z, z)} .
$$

Proof. Note

$$
\left|\delta_{z} f\right|=|f(z)|=\left|\left\langle f, K_{A}(\cdot, z)\right\rangle\right| \leq\|f\|_{\mathrm{F}_{A}} K_{A}(z, z)^{1 / 2}
$$

follows from the reproducing kernel property

$$
\left\|K_{A}(\cdot, z)\right\|_{\mathrm{F}_{A}}^{2}=\left\langle K_{A}(\cdot, z), K_{A}(\cdot, z)\right\rangle_{\mathrm{F}_{A}}=K_{A}(z, z) .
$$

This last calculation also shows that the inequality in (6.1) is an equality if $f=K_{A}(\cdot, z)$ and thereby shows that $\left\|\delta_{z}\right\|$ is actually equal to $K_{A}(z, z)^{1 / 2}$. The latter is readily checked to be equal to $c_{A}^{-1} e^{(A z, z)}$.

We have already used the first of the following two facts about $c_{A}$. 
Lemma 6.2. For the constant $c_{A}$ we have

$$
c_{A}^{-2}=\left(\frac{\operatorname{det}_{V} H}{\operatorname{det}_{V} A}\right)^{2}=\frac{\operatorname{det} T}{(\operatorname{det} S)^{1 / 2} \operatorname{det} L}
$$

where, as before, $L=(2 T-S)^{1 / 2}$ and $S=2\left(R^{-1}+T^{-1}\right)^{-1}$.

Proof. Recall from (5.3) that $2 T-S=T R^{-1} S$. Note also that

$$
S^{-1}=\frac{1}{2}\left(R^{-1}+T^{-1}\right)=R^{-1} \frac{R+T}{2} T^{-1}=R^{-1}\left(H \mid V_{\mathrm{R}}\right) T^{-1}
$$

So

$$
\begin{aligned}
& \left(\frac{\operatorname{det}_{V} A}{\operatorname{det}_{V} H}\right)^{1 / 2} \frac{\operatorname{det} T}{(\operatorname{det} S)^{1 / 2} \operatorname{det} L} \\
& \quad=\frac{(\operatorname{det} R)^{1 / 2}(\operatorname{det} T)^{1 / 2}}{\operatorname{det} S^{-1} \operatorname{det} R \operatorname{det} T} \frac{\operatorname{det} T}{(\operatorname{det} S)^{1 / 2} \operatorname{det} T^{1 / 2} \operatorname{det} R^{-1 / 2} \operatorname{det} S^{1 / 2}} \\
& =1
\end{aligned}
$$

which implies the desired result.

Next we prove a determinant relation which implies $c_{A} \geq 1$. (This "determinant AM-GM inequality" could be obtained by reference to standard matrix inequalities, but we include a complete proof.)

LEMMA 6.3. If $R$ and $T$ are positive definite $n \times n$ matrices (symmetric if real) then

$$
\sqrt{\operatorname{det} R \operatorname{det} T} \leq \operatorname{det}\left(\frac{R+T}{2}\right)
$$

with equality if and only if $R=T$.

Proof. Noting that $R^{-1 / 2} T R^{-1 / 2} \geq 0$ we have, with $D=\left(R^{-1 / 2} T R^{-1 / 2}\right)^{1 / 4}$,

$$
\begin{aligned}
\frac{\operatorname{det} R \operatorname{det} T}{\left(\operatorname{det} \frac{R+T}{2}\right)^{2}} & =\frac{\operatorname{det} R \operatorname{det}\left(R^{1 / 2} D^{4} R^{1 / 2}\right)}{\left[\operatorname{det} R^{1 / 2}\left(\frac{1+D^{4}}{2}\right) R^{1 / 2}\right]^{2}} \\
& =\left[\operatorname{det}\left(\frac{D^{2}+D^{-2}}{2}\right)\right]^{-2} \\
& =\left[\operatorname{det}\left\{I+\left(\frac{1}{\sqrt{2}} D-\frac{1}{\sqrt{2}} D^{-1}\right)^{2}\right\}\right]^{-2} .
\end{aligned}
$$


Diagonalizing $D$, it is clear that this last term is less or equal to 1 with equality if and only if $D=D^{-1}$. This is equivalent to $D^{4}=I$ which holds if and only if $R=T$.

As consequence, we have for $c_{A}$ :

$$
c_{A}=\left(\frac{\operatorname{det}_{V} A}{\operatorname{det}_{V} H}\right)^{1 / 4}=\left(\frac{\operatorname{det} R \operatorname{det} T}{\left(\operatorname{det} \frac{R+T}{2}\right)^{2}}\right)^{1 / 4}=\left(\frac{\sqrt{\operatorname{det} R \operatorname{det} T}}{\operatorname{det} \frac{R+T}{2}}\right)^{1 / 2}
$$

and so

$$
c_{A}^{-2}=\frac{\operatorname{det} \frac{R+T}{2}}{\sqrt{\operatorname{det} R \operatorname{det} T}} \geq 1
$$

with equality if and only if $R=T$.

In extending this theory to infinite dimensions, to retain a meaningful notion of evaluation $\delta_{z}: f \mapsto f(z)$, the constant $c_{A}^{-1}$, which appears in the norm $\left\|\delta_{z}\right\|$, must be finite. The expression for $c_{A}^{-2}$ in (6.2) gives a more explicit condition on $R$ and $T$ for this finiteness to hold.

If $R=r I$ and $T=t I$ then, by $(6.2), c_{A}^{-1}=[(r+t) /(2 \sqrt{r t})]^{n / 2}$ which is bounded as $n \nearrow \infty$ if and only if $r=t$ (this was noted in [13]).

\section{Remarks on extension to infinite dimensions}

The Gaussian formulation permits extension to infinite dimensions with some conditions placed on $A$. Suppose then that $V$ is an infinite-dimensional separable complex Hilbert space, $V_{\mathrm{R}}$ a real subspace on which the inner-product is real-valued and for which $V=V_{\mathrm{R}}+i V_{\mathrm{R}}$, and $A: V \rightarrow V$ a bounded symmetric, positive-definite real-linear operator carrying $V_{\mathrm{R}}$ into itself. The operators $R, T, S, H$ and $K$ are defined as before. Assume that $R$ and $T$ commute and that there is an orthonormal basis $e_{1}, e_{2}, \ldots$ of $V_{\mathrm{R}}$ consisting of simultaneous eigenvectors of $R$ and $T$ (greater generality may be possible but we discuss only this case). Let $V_{n}$ be the complex linear span of $e_{1}, \ldots, e_{n}$, and $V_{n, \mathrm{R}}$ the real linear span of $e_{1}, \ldots, e_{n}$. Then $A$ restricts to an operator $A_{n}$ on $V_{n}$, and we have similarly restrictions $H_{n}, K_{n}$ on $V_{n}$ and $R_{n}, T_{n}, S_{n}$ on $V_{n, \mathrm{R}}$. The unitary transform $S_{A}$ may be obtained as a limit of the finite-dimensional transforms $S_{A_{n}}$.

The Gaussian kernels $\rho_{S}$ and $\rho_{T}$ do not make sense anymore, and nor does the coherent state $c$, but the Gaussian measures $d \gamma_{S}(x)=\rho_{S}(x) d x$ and $\mu_{A}$ do have meaningful analogs. There is a probability space $V_{R}^{\prime}$, with a $\sigma$ algebra $\mathscr{F}$ on which there is a measure $\gamma_{S}$, and there is a linear map $V_{\mathrm{R}} \rightarrow$ $L^{2}\left(V_{\mathrm{R}}^{\prime}, \gamma_{A}\right): x \mapsto G(x)=(x, \cdot)$, such that the $\sigma$-algebra $\mathscr{F}$ is generated 
by the random variables $G(x)$, and each $G(x)$ is real Gaussian with mean 0 and variance $\left(S^{-1} x, x\right)$. Similarly, there is probability space $V^{\prime}$, with a $\sigma$ algebra $\mathscr{F}_{1}$ on which there is a measure $\mu_{A}$, and there is a real-linear map $V \rightarrow L^{2}\left(V^{\prime}, \mu_{A}\right): z \mapsto G_{1}(z)=(z, \cdot)$, such that the $\sigma$-algebra $\mathscr{F}_{1}$ is generated by the random variables $G_{1}(z)$, and each $G_{1}(z)$ is (real) Gaussian with mean 0 and variance $\frac{1}{2}\left(A^{-1} z, z\right)$. Then for each $z \in V$, written as $z=$ $a+i b$ with $a, b \in V_{\mathrm{R}}$, we have the complex-valued random variable on $V^{\prime}$ given by $\tilde{z}=G_{1}(a)+i G_{1}(b)$. Suppose $g$ is a holomorphic function of $n$ complex variables such that $\int_{V}\left|g\left(\tilde{e}_{1}, \ldots, \tilde{e}_{n}\right)\right|^{2} d \mu_{A}<\infty$. Define $\mathrm{F}_{A}$ to be the closed linear span of all functions of the type $g\left(\tilde{e}_{1}, \ldots, \tilde{e}_{n}\right)$ in $L^{2}\left(\mu_{A}\right)$ for all $n \geq 1$. We may then define $S_{A}$ of a function $f\left(G\left(e_{1}\right), \ldots, G\left(e_{n}\right)\right)$ to be $\left(S_{A_{n}} f\right)\left(\tilde{e}_{1}, \ldots, \tilde{e}_{n}\right)$, and then extend $S_{A}$ by continuity to all of $L^{2}\left(\gamma_{S}\right)$. In writing $S_{A_{n}} f$ we have identified $V_{n}$ with $C^{n}$ and $V_{n, \mathrm{R}}$ with $\mathrm{R}^{n}$ using the basis $e_{1}, \ldots, e_{n}$.

A potentially significant application of the infinite-dimensional case would be to situations where $V_{\mathrm{R}}$ is a path space and $A$ arises from a suitable differential operator. For the "classical case" where $R=T=t I$ for some $t>0$, this leads to the Hall transform [6] for Lie groups as well as the path-space version on Lie groups considered in [8].

\section{REFERENCES}

1. Bargmann, V., On a Hilbert space of analytic functions and an associated integral transform, Part I, Comm. Pure. Appl. Math. 14 (1961), 187-214.

2. Driver, B., and Hall, B., Yang-Mills theory and the Segal-Bargmann transform, Comm. Math. Phys. 201 (1999), 249-290.

3. Fock, V., Verallgemeinerung und Lösung der Diracschen statistischen Gleichung, Z. für Phys. 49 (1928), 339-357.

4. Fock, V., Konfigurationsraum und zweite Quantelung, Z. für Phys 75 (1932), 622-647.

5. Folland, G. B., Harmonic Analysis in Phase Space, Ann. of Math. Stud. 122 (1989).

6. Hall, B. C., The Segal-Bargmann "coherent state” transform for compact Lie groups, J. Funct. Anal. 112 (1994), 103-151.

7. Hall, B. C., The range of the heat operator, Preprint, 2004.

8. Hall, B. C., and Sengupta, A. N., The Segal-Bargmann transform for path spaces in groups, J. Funct. Anal. 152 (1998).

9. Ólafsson, G., Analytic continuation in representation theory and harmonic analysis, in J. P. Bourguignon, T. Branson, and O. Hijazi (eds.), Seminares et Congr. Vol. 4 (2000), 201233, The French Math. Soc.

10. Ólafsson, G., and Ørsted, B., Generalizations of the Bargmann transform, Lie theory and its applications in physics, Clausthal 1995 (H.-D. Doebner, V. K. Dobrev and J. Hilgert, eds.), World Scientific, River Edge, New Jersey, 1996.

11. Segal, I. E., Foundations of the theory of dynamical systems of infinitely many degrees of freedom I, Mat. Medd. Danske Vid. Selsk. 31 (1959), no. 12.

12. Segal, I. E., Mathematical Problems of Relativistic Physics, Amer. Math. Soc., 1963. 
13. Sengupta, A. N., The two-parameter Segal-Bargmann transform, Preprint (1999) at http://www.mpim-bonn.mpg.de/html/preprints/preprints.html.

DEPARTMENT OF MATHEMATICS

LOUISIANA STATE UNIVERSITY

BATON ROUGE, LA 70803

USA

E-mail: fabec@math.lsu.edu, olafsson@math.lsu.edu,sengupta@math.lsu.edu

URL: www.math.lsu.edu/ fabec, www.math.lsu.edu/ ${ }^{\sim}$ olafsson, www.math.lsu.edu/ $\sim$ sengupta 